\title{
On M-theory on Real Toric Fibrations
}

\author{
A. Belhaj ${ }^{1}$, H. Belmahi ${ }^{1}$, M. Benali ${ }^{1}$, S-E. Ennadifi ${ }^{2}$, Y. Hassouni $^{1}$, Y. Sekhmani ${ }^{1 *}$ \\ ${ }^{1}$ Equipe des Sciences de la matière et du rayonnement, ESMaR, Département de Physique \\ Faculté des Sciences, Université Mohammed V de Rabat, Morocco \\ ${ }^{2}$ LHEP-MS, Département de Physique, Faculté des Sciences \\ Université Mohammed V de Rabat, Morocco
}

June 21, 2021

\begin{abstract}
Borrowing ideas from elliptic complex geometry, we approach M-theory compactifications on real fibrations. Precisely, we explore real toric equations rather than complex ones exploited in F-theory and related dual models. These geometries have been built by moving real toric manifolds over real bases. Using topological changing behaviors, we unveil certain data associated with gauge sectors relying on affine Lie symmetries. Keywords: Torus moduli; compactifications; real fibrations; Higher dimensional theories; D-branes; Lie symmetries.
\end{abstract}

*Authors in alphabetical order. 


\section{Introduction}

Complex algebraic geometry has been considered as a crucial tool not only in mathematics but also in physics [1]. In particular, it has been used in modern physics and related topics. It concerns building of compact complex manifolds needed for string compactification mechanisms [2]. A special emphasis has been put on Calabi-Yau geometries supported by the industry of stringy models providing certain semi-realistic theories going beyond standard models. Among others, the elliptic fibrations of Calabi-Yau manifolds have been extensively investigated in connection with F-theory and stringy dual models. It is recalled that F-theory, which is a 12-dimensional theory, was proposed after the discovery of dualities between superstring theories in 10-dimensions [2]. This theory has been considered as an alternative way to approach the strong coupling limits of certain superstring theories from higher dimensions being not discovered in M-theory scenarios [3]. Exploiting the $S L(2, \mathbb{Z})$ symmetry, the compactification of such a theory on the torus $\mathbb{T}^{2}$ has been studied. In a specific limit of the $\mathbb{T}^{2}$ moduli space, this can produce type IIB superstring theory in 10dimensions [2]. This has been established by identifying the $\mathbb{T}^{2}$ shape complex moduli with a complex scalar field formed by combining the axion $\chi$ and the dilaton $\phi$ fields. Lower dimensional F-theory models have been built using the elliptic fibrations on complex base manifolds, providing many supersymmetric vacuum solutions $[4,5]$. In particular, the associated gauge theories have been geometrically engineered from complex elliptic fibrations of Calabi-Yau manifold spaces [6-10]. However, the torus involves another parameter controlling its size which has been frozen in F-theory building models. This size has been frozen because it is not invariant under $S L(2, \mathbb{Z})$ symmetry describing the self duality of type IIB superstring. In such activities, it has been considered as a non dynamical quantity. At this level, one could naturally ask certain questions about such a real parameter using elliptic real fibrations in stringy compactification scenarios.

The aim of this work is to mimetic the complex elliptic fibrations by introducing toric fibrations relying on a real algebraic geometry. A close inspection shows that M-theory could be considered as a possible candidate where real toric fibrations could find a place. Concretely, we first explore equations describing real fibration manifolds, rather than complex ones exploited either in F-theory or in dual theories. Then, we study the M-theory compactification on such real fibration geometries and propose a conjecture in terms of heterotic superstring models. These geometries have been constructed by moving real toric manifolds over real bases. Using topological changing behaviors, we unveil certain data associated with gauge sectors relying on affine Lie symmetries.

The organization of this work is as follows. In section 2, we deal with M-theory on $\mathbb{T}^{2}$ using a real algebraic geometry. In section 3, we explore real toric fibrations to approach a Mtheory compactification down to eight dimensions and make contact with Higgising matter contents. Section 4 concerns an extended compactification of such real elliptic fibrations. The last section is devoted to concluding remarks and conclusions. 


\section{$2 \quad$ M-theory on real torus}

In this section, we investigate M-theory on a real algebraic geometry of $\mathbb{T}^{2}$. At low energies, this theory is described by eleven dimensional supergravity involving certain bosonic fields coupled to M-branes. Before going ahead, it is recalled that the moduli space of $\mathbb{T}^{2}$ involves two different kinds of parameters which control the shape and the size associated with the metric deformations. A nice way to understand such a moduli space is to exploit string theory compactifications. Indeed, one uses the following parameter space

$$
\mathcal{M}\left(\mathbb{T}^{2}\right)=\frac{S L(2)}{U(1)} \times \frac{S L(2)}{U(1)}
$$

where the first factor $\frac{S L(2)}{U(1)}$ can be viewed as the complex structure and the other one corresponds to the Kähler structure and the stringy B-field. Removing such a B-field, the reduced factor corresponds to the size deformation, being frozen in certain elliptic CalabiYau compcatifiactions relying on the complex geometry [4-8]. Here, however, we would like to elaborate real toric fibration compactifications in M-theory by combining the real region of the $\mathbb{T}^{2}$ moduli space and real toric geometry bundles. Indeed, an examination shows that $\mathbb{T}^{2}$ involves a real algebraic representation given by the following equation

$$
\left(R-\sqrt{x_{2}^{2}+x_{3}^{2}}\right)^{2}+x_{1}^{2}=r^{2},
$$

where $x_{1}, x_{2}$ and $x_{3}$ are real coordinates of the 3 -dimensional non compact flat space [11]. $R$ and $r$ are real parameters corresponding to the involved fibred circles having different lengths with the condition $R>r$ needed to provide a torus manifold. For $R=2$ and $r=1$, this real geometry is illustrated in figure 1 . It is noted that $R$ and $r$ are now real

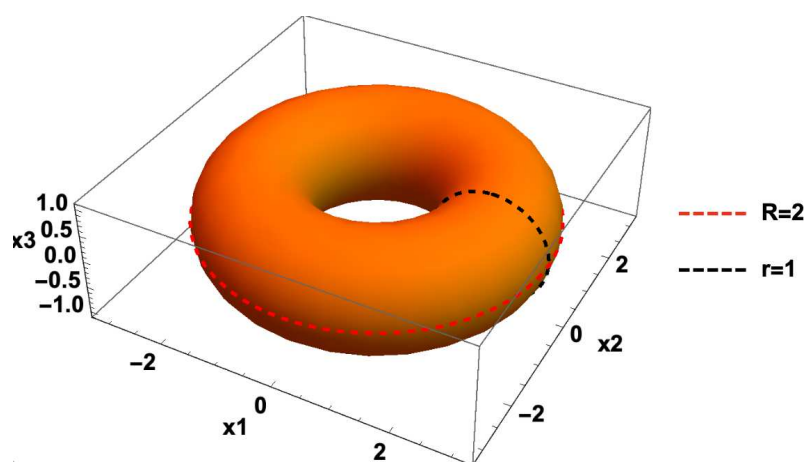

Figure 1: Graphic representation of two dimensional torus for $R=2$ and $r=1$.

parameters controlling the area of $\mathbb{T}^{2}$. This geometry can be parameterized by the following real relations

$$
\begin{aligned}
& x_{1}=r \sin \theta_{1}, \\
& x_{2}=\left(R+r \cos \theta_{1}\right) \sin \theta_{2}, \\
& x_{3}=\left(R+r \cos \theta_{1}\right) \cos \theta_{2},
\end{aligned}
$$


where $\theta_{1}$ and $\theta_{2}$ are the toric coordinates of the torus constrained by $0 \leq \theta_{i} \leq 2 \pi(i=1,2)$. In this geometry, the metric line element can be written as

$$
d s^{2}=g_{a b} d \theta^{a} d \theta^{b}, \quad a, b=1,2 .
$$

This metric $g_{a b}$ is given by

$$
g_{a b}=\sum_{i, j=1}^{3} \delta_{i j} \frac{\partial x^{i}}{\partial \theta^{a}} \frac{\partial x^{j}}{\partial \theta^{b}}=\left(\begin{array}{cc}
r^{2} & 0 \\
0 & \left(R+r \cos \theta_{1}\right)^{2}
\end{array}\right), \quad i, j=1,2,3,
$$

and where $\delta_{i j}$ is the Kronecker symbol. The area of $\mathbb{T}^{2}$ can be computed using the following integration equation

$$
A\left(\mathbb{T}^{2}\right)=\iint_{\mathbb{T}^{2}} \sqrt{|g|} d \theta_{1} d \theta_{2}
$$

where $g$ is the determinant of the induced metric. Using the equation (2.6), one obtains

$$
A\left(\mathbb{T}^{2}\right)=\int_{0}^{2 \pi} d \theta_{1} \int_{0}^{2 \pi} r\left(R+r \cos \theta_{1}\right) d \theta_{2}=4 \pi^{2} r R .
$$

It follows that this real analysis of $\mathbb{T}^{2}$ involves only size parameter contributions controlling the area. It is clear that this road does not provide any complex scalar field in M-theory compactifications. However, it could give only a real scalar field which can be identified with the size of $\mathbb{T}^{2}$. A close inspection shows that one can consider the dilaton scalar filed as a possible candidate. Forgetting about the full desired spectrum of the compactified M-theory, it has been observed that $R$ and $r$ should be considered as relevant parameters to approach such a field content in lower dimensions. We expect that these lower compactifications could bring extra information on such real toric fibrations in the compactification of Mtheory. By mimicking the analysis explored in the complex algebraic geometry of F-theory stringy models, we attempt to investigate the involved compactifications by varying the size parameters of $\mathbb{T}^{2}$ over real base manifolds, rather than complex bases exploited in known higher dimensional theories, including superstringy models and F-theory.

\section{Eight dimensional models}

Borrowing ideas from known complex compactifications, the lower dimensional models from real toric fibrations in compactifications of M-theory can be obtained by varying circle length parameters over $d$-dimensional real bases by freezing the shape parameter of $T^{2}$ corresponding to the first factor of $M\left(T^{2}\right)$. This could give models in $(9-d)$ dimensions.

To see how this works in practice, we deal with the case $d=1$ associated with the circle and the interval compactifications. However, to get a non-trivial geometry and motivated by stringy known results including Hořava-Witten models, we deal with only an interval compactification. A priori, there are many ways to construct the resulting desired geometry. A way is to take the following real algebraic fibration equation

$$
\left(R(\eta)-\sqrt{x_{2}^{2}+x_{3}^{2}}\right)^{2}+x_{1}^{2}=r(\eta)^{2}
$$


where $\eta$ indicates the coordinate of the interval real base being constrained by $0 \leq \eta \leq \pi$. It is recalled that this real base space geometry could be built from a circle $S^{1}$ defined by

$$
\eta=\eta+1
$$

up to a $Z_{2}$ orbifold symmetry acting as follows

$$
\eta \rightarrow-\eta
$$

Roughly speaking, non-trivial real fibrations can be handled by considering generic length functions. However, we expect that periodicity, linearity and quadratic functions are useful mathematical features providing simple tools to visualize such real toric fibrations. To make contact with known models, we propose the following specific variations

$$
\begin{aligned}
R(\eta) & =R \sin \eta, \\
r(\eta) & =r .
\end{aligned}
$$

In this way, the equation (2.3) becomes

$$
\begin{aligned}
& x_{1}(\eta)=r \sin \theta_{1}, \\
& x_{2}(\eta)=\left(R \sin \eta+r \cos \theta_{1}\right) \sin \theta_{2}, \\
& x_{3}(\eta)=\left(R \sin \eta+r \cos \theta_{1}\right) \cos \theta_{2} .
\end{aligned}
$$

The associated geometry for specific points on the interval is illustrated in figure 2 . It
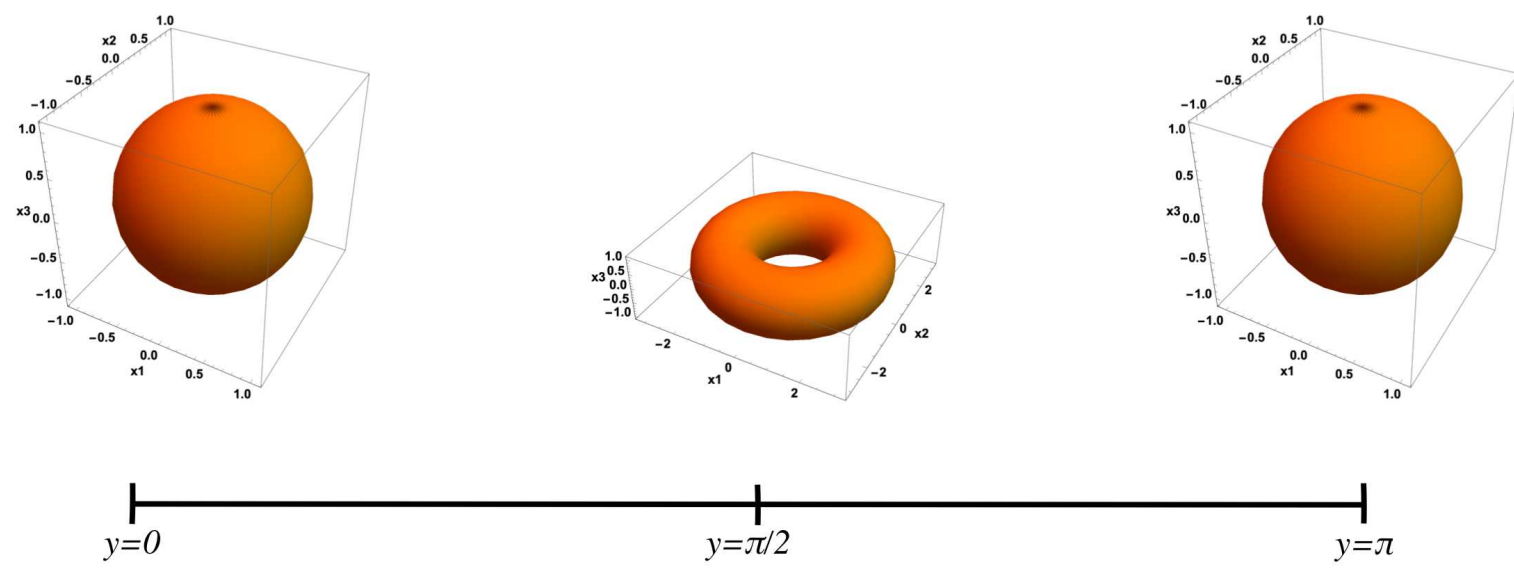

Figure 2: Fibration behaviors on the interval $[0, \pi]$.

is remarked that a real 2-sphere appears at the end of the interval $\eta=0$ and $\eta=\pi$ parameterized by

$$
\begin{aligned}
x_{1}(\eta) & =r \sin \theta_{1}, \\
x_{2}(\eta) & =r \cos \theta_{1} \sin \theta_{2}, \\
x_{3}(\eta) & =r \cos \theta_{1} \cos \theta_{2}, \\
R(\eta) & =0 .
\end{aligned}
$$


Its area can be calculated from the following tensor metric

$$
g_{a b}=\left(\begin{array}{cc}
r^{2} & 0 \\
0 & r^{2} \cos ^{2} \theta_{1}
\end{array}\right) .
$$

Indeed, it is given by

$$
A\left(\mathbb{S}^{2}\right)=\iint_{\mathbb{S}^{2}} \sqrt{|g|} d \theta_{1} d \theta_{2}=4 \pi r^{2}
$$

It follows from figure 2 that $\mathbb{T}^{2}$ changes its geometry producing non-trivial fibers over the real interval. We will see that the area of such a real fibration is a function of the base coordinate $\eta$. Similar ideas, in connections with material physics, have been discussed in [12]. Concretely, the area can be obtained via the following relation

$$
A(\eta)=\int_{0}^{2 \pi} \int_{-\pi+u(\eta)}^{\pi-u(\eta)} \sqrt{|g(\eta)|} d \theta_{1} d \theta_{2}
$$

where $g(\eta)$ is now the determinant of the fibration metric

$$
g_{a b}(\eta)=\left(\begin{array}{cc}
r^{2} & 0 \\
0 & \left(R \sin \eta+r \cos \theta_{1}\right)^{2}
\end{array}\right) .
$$

It is noted that the function $u(\eta)$ has been introduced as a generic function carrying information of the $\theta_{1}$ dependence of the fibration. This idea has been inspired by the torus parametrization showing that $R$ and $r \cos \theta_{1}$ should be treated on an equal footing. After integral calculi, we obtain the following varying function area

$$
A(\eta)=2 \pi\left[R r \sin \eta(2 \pi-2 u(\eta))+2 r^{2} \sin u(\eta)\right]
$$

A simple observation shows that this area splits into two different parts

$$
A(\eta)=a_{1}(\eta) A\left(\mathbb{T}^{2}\right)+a_{2}(\eta) A\left(\mathbb{S}^{2}\right)
$$

where $A\left(\mathbb{T}^{2}\right)$ and $A\left(\mathbb{S}^{2}\right)$ are the area of $\mathbb{T}^{2}$ and $\mathbb{S}^{2}$, respectively. However, $a_{1}(\eta)$ and $a_{2}(\eta)$ are real fibration functions given by

$$
\begin{aligned}
& a_{1}(\eta)=\frac{\sin \eta}{\pi}(\pi-u(\eta)) \\
& a_{2}(\eta)=\sin u(\eta) .
\end{aligned}
$$

An examination reveals that the variation of the fiber appearing in figure 2 can be assured by the following fibration function

$$
u(\eta)=\arccos (\sin \eta)
$$

From the figure 2, we observe that the fibration generates a smoothness breakdown corresponding to the appearance of a real 2-sphere at the ends of the interval real base given by 
$\eta=0$ and $\eta=\pi$. This produces two different relevant topological behaviors. The first one is singular associated with the limits

$$
\begin{aligned}
R(\eta) & \rightarrow 0 \\
a_{1}(\eta) & \rightarrow 0 \\
A(\eta) & \rightarrow A\left(\mathbb{S}^{2}\right),
\end{aligned}
$$

occurring at the ends of the interval $\eta=0, \pi$. While, the second one is a smooth torus behavior corresponding to

$$
\begin{aligned}
R(\eta) & \rightarrow R \\
a_{2}(\eta) & \rightarrow 0, \\
A(\eta) & \rightarrow A\left(\mathbb{T}^{2}\right),
\end{aligned}
$$

being localized at the middle of the interval $\eta=\frac{\pi}{2}$. These behaviors relay on the Euler characteristic $\chi\left(\mathbb{T}^{2}\right)=0$ and $\chi\left(\mathbb{S}^{2}\right)=2$, respectively. It is recalled that the Euler characteristic appears in many places in string theory and related topics including Calabi-Yau compactifications. Among others, it takes places in intersecting geometries used in the brane realization of gauge theories from complex algebraic compactifications [13]. For certain Calabi-Yau manifolds, these intersecting geometries are linked to Lie symmetries [14]. Based on this remark, the above singular behaviors could be worked out to supply possible connections with certain known theories up to some assumptions.

Similarities with such theories push one to unveil hidden sectors. Indeed, singular behaviors of such real fibrations could be linked to Lie symmetries. This road deserves more ideas of reflections. It is worth noting that many interesting results in string theory and related models have been discovered from the metric and the topological properties of compact manifolds using the geometric engineering method [15]. Motivated by such activities, we could bring certain information on the gauge sector. This invisible sector could be approached by combining certain topological and geometrical pictures associated with singular behaviors, the Euler characteristic changing, and the torus behaviors in terms of gluing spheres from a weighted sum. To reach the goal that we are looking for, we engineer the fiber as a 2dimensional real space $\mathbb{M}^{2}$ by gluing $(n+1)$ real 2-spheres. The adjacent 2-spheres intersect on a disk. However, we consider the limit where such a disk shrinks to a point. This geometrical configuration has been extensively studied in connections with material physics. Motivated by weighted sizes explored in such a physics [16], we represent the manifold $\mathbb{M}^{2}$ by a weighted connected sum of $(n+1)$ real 2 -spheres given by

$$
\mathbb{M}^{2}=\sum_{p=0}^{n} \omega_{p} \mathbb{S}_{p}^{2}
$$

where $\omega_{p}$ is the weight of $p$-th 2 -sphere. A simple configuration is illustrated in figure 3 , by gluing only two 2-spheres. 


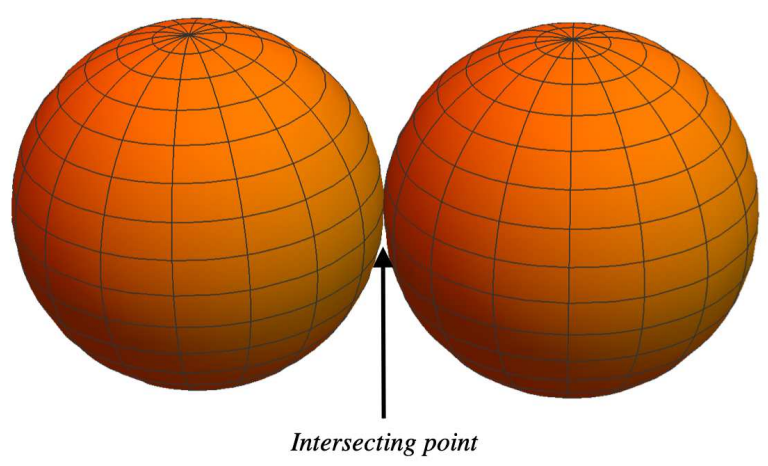

Figure 3: Gluing spheres.

According to many works including $[17,18]$, it is noted that the self intersection of $\mathbb{M}^{2}$ is linked to the Euler characteristic via the relation

$$
\chi\left(\mathbb{M}^{2}\right)=-\mathbb{M}^{2} \cdot \mathbb{M}^{2} .
$$

Using the equation(3.19), this can be re-written as

$$
\chi\left(\mathbb{M}^{2}\right)=-\sum_{q=0}^{n} \sum_{p=0}^{n} \omega_{q} \omega_{p} I_{p q}
$$

where $I_{p q}$ denotes the intersection number between $\mathbb{S}_{p}^{2}$ and $\mathbb{S}_{q}^{2}$

$$
I_{p q}=\mathbb{S}_{p}^{2} \mathbb{S}_{q}^{2}
$$

The vanishing of the Euler characteristic, $\chi\left(\mathbb{M}^{2}\right)=0$, corresponding to the torus behavior, is assured by the following condition

$$
\sum_{q=0}^{n} \omega_{q}\left(\sum_{p=0}^{n} \omega_{p} I_{p q}\right)=0 .
$$

For generic $\omega_{q}$ weights, this condition can be solved by

$$
\sum_{p=0}^{n} \omega_{p} I_{p q}=0
$$

It is noted that this constraint shears similarities with the toric realisation of local CalabiYau three-folds with elliptic singularities. In particular, this can represent the compact part used to remove the associated singularities where the entries of the matrix $I_{p q}$ provide the intersection numbers of the involved blowing up cycles $[8,9]$ A priori, there could be many other ways to verify such a condition. However, a possible one is to exploit results explored in the theory of Lie symmetries [19]. Indeed, we propose the following solution

$$
\begin{aligned}
I_{p q} & =-K_{p q}, \\
\omega_{p} & =\delta_{p},
\end{aligned}
$$


where $K_{p q}$ and $\delta_{p}$ are the Cartan matrices and the Dynkin weights of affine Lie symmetries, respectively. In this context, $n$ is identified with the involved rank. An examination shows that the matrix $I_{p q}$ should be symmetric which requires a particular choice for such symmetries. They are identified with affine simply laced ones. The graphic representation of these symmetries are given in figure 4 .

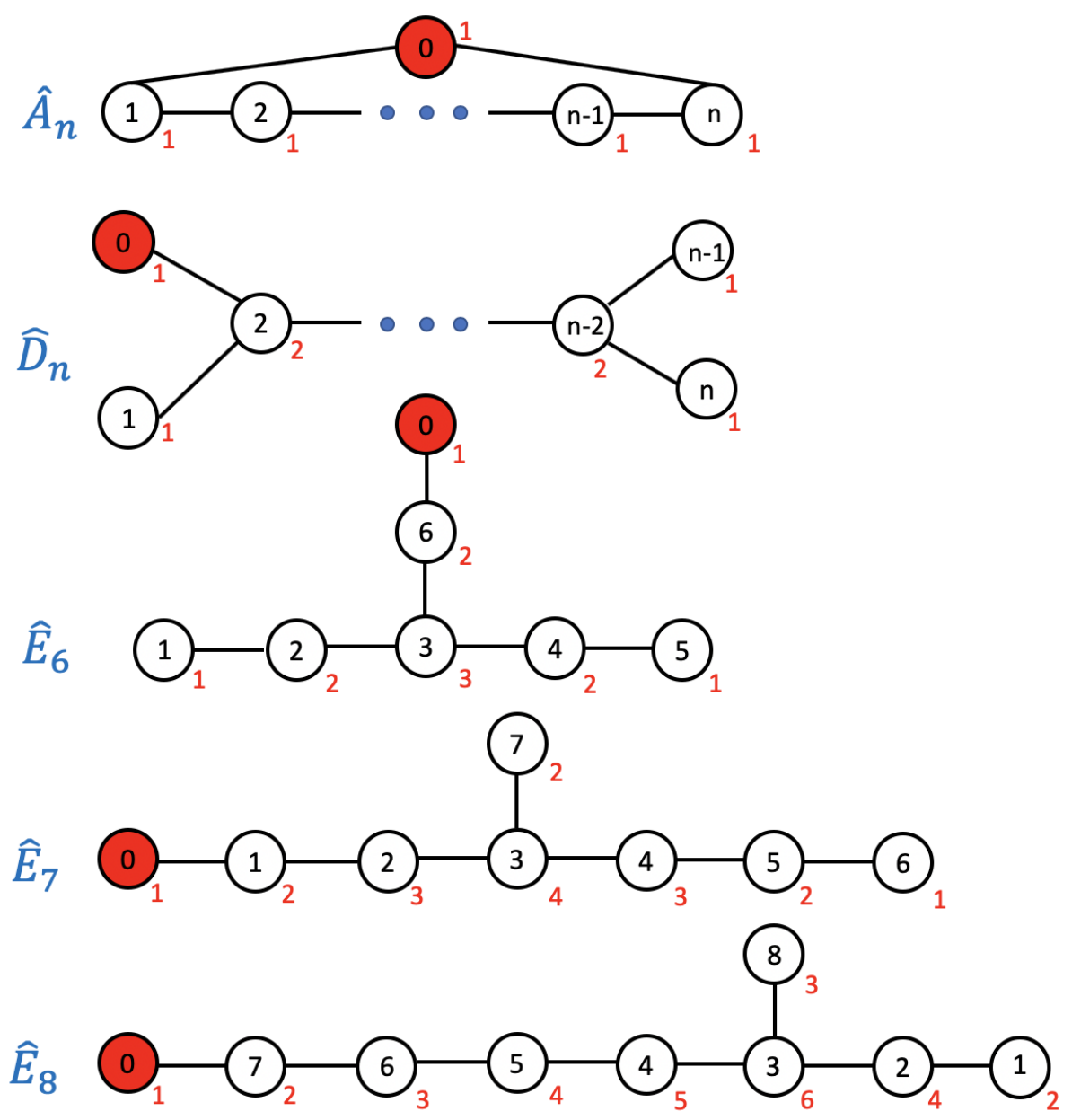

Figure 4: ADE affine Lie symmetries with Dynkin weights given in red color. The red vertex represents the affine one.

In the present context, the above sum can be reduced to

$$
\mathbb{M}^{2}=\mathbb{S}_{0}^{2}+\sum_{p=1}^{n} \delta_{p} \mathbb{S}_{p}^{2}
$$

where we have taken $\delta_{0}=1$ which corresponds to the affine root. However, the remaining values of $\delta_{p}(p \neq 0)$ fix the desired affine Lie symmetry. In this language, the torus behavior is mapped to the imaginary root of such a Lie symmetry given by

$$
\beta=\alpha_{0}+\sum_{p=1}^{n} \delta_{p} \alpha_{p}
$$


where $\alpha_{p}$ are the involved simple roots. In this geometric picture, the first 2 -sphere $\mathbb{S}_{0}^{2}$ corresponds to the affine simple root which will carry singular behavior information. Indeed, the area of $\mathbb{M}^{2}$ takes the following form

$$
A\left(\mathbb{M}^{2}\right)=4 \pi\left(r_{0}^{2}+\sum_{p=1}^{n} \delta_{p} r_{p}^{2}\right)
$$

where now $\delta_{p}$ can be regarded as radius scaling factors. It is remarked that the torus behavior appearing at the middle of the interval corresponds to the following size constraints

$$
\begin{aligned}
& r_{0} \neq 0, \\
& r_{p} \neq 0 \quad p=1, \ldots, n .
\end{aligned}
$$

However, these real parameters should satisfy the following relation

$$
r_{0}^{2}+\sum_{p=1}^{n} \delta_{p} r_{p}^{2}=R r .
$$

In this way, the singular behaviors located at the interval ends $(y=0$ and $y=\pi)$ are associated with

$$
\begin{aligned}
& r_{p}=0 \quad p=1, \ldots, n, \\
& r_{0}=r .
\end{aligned}
$$

Identifying such real parameters with the mass of the involved modes, this singular limit could generate massless ones. In this way, the $E_{8}$ symmetry factor appearing in many stringy models can be approached by taking the following choice of weighted radius parameters

$$
\delta_{0}=1, \quad \delta_{1}=2, \quad \delta_{2}=4, \quad \delta_{3}=6, \quad \delta_{4}=5, \quad \delta_{5}=4, \quad \delta_{6}=3, \quad \delta_{7}=2, \quad \delta_{8}=3 .
$$

This can give rise to Higgsing field contents in M-theory compactifications providing a possible geometrical evidence for the existence of certain gauge theories relying on real toric fibrations. Indeed, according to such a basic representation, the Dynkin weights correspond to simple roots of the algebra. Removing a node from the extended Dynkin diagram, it can be viewed as the reducing symmetry pattern in accord with adjoint Higgsing. Roughly, for the Dynkin diagrams representation of the $E_{n}$ Lie algebras, the Higgsing i.e., the corresponding symmetry breaking $E_{n} \rightarrow E_{n-1}$ can be pictured as the suppression of a particular node from the $E_{n}$ Dynkin diagram [20-22]. The depiction that has to be employed for the Higgsing is the one with the suppressed node, whose the highest weight is equal to its corresponding root. This could be approached using quiver gauge theories based on affine Dynkin diagrams.

An examination reveals that one could anticipate a possible conjectured duality formulated as follows

$$
\text { M-theory on } \mathbf{R}^{1,8} \times \mathbb{T}^{2} \times[0, \pi] \longrightarrow \text { Heterotic theory on } \mathbf{R}^{1,7} \times \mathbb{T}^{2}
$$


where one circle of heterotic string theory is identified with the non-vanishing circle of Mtheory real torus. One could provide certain arguments in favor of this anticipated mapping duality. At first sight, this duality seems a little bit different since generally one exploits complex structures to approach certain dualities associated with F-theory compactifications. However, here, we are implementing real parameters. The latters, which have been not explored, could unveil new data on the anticipated duality. In addition to this, the adiabatic argument could also bring extra supports [23]. Since M-theory on $\mathbb{T}^{2}$ is equivalent to type IIB on $S^{1}$, we can compactify both sides on the interval by varying the size parameters. This could be linked to type I superstring [24]. In certain limits, the latter is dual to heterotic string in 9-dimensions. Based on these arguments, one should expect that there could be such a conjectured duality as the one appearing in F-theory relying on shape parameters. Forgetting about other arguments, this disinterest could be due to the lack of mathematical results associated with real fibrations. This needs more reflections which are left for future works.

\section{Extended compactification models}

In this section, we would like to generalize the previous finding. Concretely, we consider a generalized real toric fibration. A close inspection shows that the previous real toric fiber can be extended as follows

$$
S^{1} \times S^{1} \longrightarrow \mathcal{T}^{n}=S^{1} \times S^{n-1} .
$$

where $S^{1}$ will be replaced by $S^{n-1}$. It is worth noting that the real toric geometry $S^{1} \times S^{n-1}$ has been approached in connection with black ring solutions [25]. A special emphasis has been put on $n=3$ associated with black rings in five dimensions considered as an intermediate solution between black holes and black strings [26]. Roughly speaking, $\mathcal{T}^{n}=S^{1} \times S^{n-1}$ can be described by the following algebraic equation

$$
\left(R-\sqrt{x_{n+1}^{2}+x_{n}^{2}}\right)^{2}+\sum_{i=1}^{n-1} x_{i}^{2}=r^{2},
$$

where one recovers (3.1) by taking $n=2$. In this way, $\mathcal{T}^{n}$ reduces to $\mathbb{T}^{2}$. An examination reveals that the above parametrization could be generalized in different ways depending on the fibration of $S^{1}$ over the base geometry $S^{n-1}$. In the generic fibration, the size of the fiber $S^{1}$ should be, in principle, a function of the local coordinates of $S^{n-1}$ and its size parameters as follows

$$
R=R\left(r, \theta_{1}, \ldots, \theta_{n-1}\right),
$$

constrained by the toric real geometry (4.2). For $n>2$, we could deal with two relevant configurations. First, we consider a simple fibration, where the fiber depends only on one local coordinate of $S^{n-1}$. In this way, the fiber size can take the following form

$$
R=R+f_{s}\left(r, \theta_{\ell}\right) .
$$


The second relevant configuration corresponds to a generic fibration function depending on all local coordinates of $S^{n-1}$ and its size parameters. In this case, we can write

$$
R=R+f_{g}\left(r, \theta_{i}\right), \quad i=1,2, \ldots, n-1 .
$$

In what follows, we will be interested in the second situation being a generic one. In particular, we propose the following parametrization

$$
\begin{aligned}
x_{1}= & r \cos \theta_{1}, \\
& \vdots \\
x_{n-1}= & r \cos \theta_{n-1} \sin \theta_{1} \ldots \sin \theta_{n-2}, \\
x_{n}= & \left(R+r \sin \theta_{1} \ldots \sin \theta_{n-1}\right) \cos \theta_{n}, \\
x_{n+1}= & \left(R+r \sin \theta_{1} \ldots \sin \theta_{n-1}\right) \sin \theta_{n},
\end{aligned}
$$

where we have taken $f_{g}$ as

$$
f_{g}\left(r, \theta_{i}\right)=f_{g}=r \prod_{i=1}^{n-1} \sin \theta_{i} .
$$

It is worth noting that for $n=2$ the equation (3.6) can be recovered by shifting $\theta_{1}$ as follows

$$
\theta_{1} \longrightarrow \theta_{1}-\frac{\pi}{2}
$$

In M-theory compactifications down to eight dimensions, the geometry $\mathbb{T}^{2} \times I$ will be replaced by $\mathcal{T}^{n} \times I$ where the size parameter $R$ is replaced, as before, by $R \sin \eta$. It is remarked that at the end of the interval similar singular behaviors appear associated with the existence of $n$-dimensional sphere $S^{n}$ with radius $r$. A connection with Lie symmetries requires certain constraints on such compactifications. Indeed, it is recalled that the Euler caracteristic of $S^{n}$ is given by

$$
\chi\left(S^{n}\right)=1+(-1)^{n} .
$$

Diagonal elements of the Cartan matrices required by

$$
\chi\left(S^{n}\right)=S^{n} \times S^{n}=2,
$$

being the self intersection, impose that $n$ should be even

$$
n=2 m \text {. }
$$

In the singular behaviors of the M-theory compactification, $S^{1} \times S^{2 m-1}$ can be viewed as a collection of $S^{2 m}$ according to affine Dynkin diagrams providing similar findings obtained in the previous section. 


\section{Concluding remarks and open questions}

The moduli space of $\mathbb{T}^{2}$ involves two different kinds of parameters which control the corresponding shape and size. The shape deformations have been extensively used in F-theory complex analysis by ignoring the other part of the moduli space associated with size deformations. Motivated by such activities, we have investigated M-theory compactifications from the size region of such a moduli space. Concretely, we have presented a road to approach M-theory compactifications relying on real parameters controlling the size of internal spaces. Concretely, we have implemented a real algebraic geometry of $\mathbb{T}^{2}$ in order to explore the physics associated with the size contributions. In particular, we have provided certain compactifications from real fibrations over real bases. Precisely, we have discussed, in some detail, eight dimensions obtained from the interval compacatification showing two different relevant behaviors. In particular, we have revealed that the middle of such an interval is associated with the torus geometrical behaviors, while the ends correspond to singular ones, discussed in terms of the Euler characteristic changing over the real base. Examining such behaviors, we have approached the gauge Higgsing content by combining many results explored in string theory and related models.

This work comes up with many remarks and open questions related to such real fibration compactifications. Assuming its existence, one natural question is to establish possible links with certain limits of known supergravity theories using duality symmetries. We anticipate to stress that there could be some links with theories based on real analysis in M-theory compactfications including G2 manifolds. Moreover, it is worth noting that such geometrical features and tools of real torus fibrations could be used in the explicit building of models with a gauge group and spectrum resembling that of the Standard Model after possible compactification scenarios [27]. Principally, the most difficult question that the stringyinspired phenomenology encounters resides in the appropriate compactification manifolds. In fact, it is just the topology of such a manifold that yields important information about the the low-energy physics, i.e. the number of supersymmetries and the fields content that survive the compactification [28]. In connection with the main theme in this work concerned with real toric fibrations, one has to deal with a geometric description of elliptically fibered spaces where a higher dimensional manifolds with only free size deformation parameters. A concrete view of the relevance of such a geometry appears in intersecting D-brane model buildings. Especially, realizations in orientifold compactifications of type II string theory with D-branes where the extra dimensions are wrapped on certain homology cycles in real toric fibrations, being the simple one where the homology cycles are easily viewable giving a non-trivial physics. Actually, it is the rich vocabulary such as intersection numbers $I$, intersection angles $\varphi_{i}$, cycles $\pi_{i}$ and winding numbers $\left(n_{i}, m_{i}\right)$, between the toric real geometry and the effective field theory existing in any D-brane model that is behind the purposes for the prominence of D-brane physics $[29,30]$. We thus believe that the corresponding realistic physics from real torus fibrations still merits a careful and deep analysis. We hope to address such questions in future investigations. 


\section{Acknowledgements}

The authors would like to thank M. P. Garcia del Moral, A. Marrani and E. Torrente Lujano for discussions on related topics and correspondence. They thank also the anonymous referee for its careful reading of the present manuscript, insightful comments, and suggestions, improving this work significantly. This work is partially supported by the ICTP through $\mathrm{AF}$.

\section{References}

[1] U. Lindstrom, R. Minasian, A. Tomasiello, M. Zabzine, Generalized complex manifolds and supersymmetry, Commun.Math.Phys. 257 (2005) 235, hep-th/0405085.

[2] C. Vafa, Evidence for F-theory, Nucl. Phys. B469 (1996)403, hep-th/9602022.

[3] E. Witten, String theory dynamics in various dimensions, Nucl. Phys. B443 (1995)85, hep-th/9503124.

[4] D. Morrison, C. Vafa, Compactifications of F-Theory on Calabi-Yau Threefolds -I, Nucl.Phys. B473 (1996)74, hep-th/9602114.

[5] D. Morrison, C. Vafa, Compactifications of F-Theory on Calabi-Yau Threefolds -II, Nucl.Phys. B476 (1996)437, hep-th/9603161.

[6] M. Bershadsky, A. Johansen, T. Pantev, V. Sadov, C. Vafa, F-theory, Geometric Engineering and $N=1$ Dualities, Nucl.Phys. B505 (1997) 153, hep-th/9612052.

[7] S. Aspinwall, S. Katz, D. Morrison, Lie Groups, Calabi-Yau Threefolds, and F-Theory, Adv. Theor. Math. Phys. 4 (2000)95, hep-th/0002012.

[8] A. Belhaj, E.H Saidi, Toric Geometry, Enhanced non Simply laced Gauge Symmetries in Superstrings and F-theory Compactifications, hep-th/0012131.

[9] S. Katz, P. Mayr and C. Vafa, Mirror symmetry and exact solution of $4 d$ N=2 gauge theories I, Adv, Theor. Math. Phys 1(1998)53, hep-th/9706110.

[10] R. Donagi, M. Wijnholt, Model Building with F-Theory, Adv.Theor.Math.Phys. 15 (2011)1237, arXiv:0802.2969.

[11] L. Moroni, The toric sections: a simple introduction, arXiv:1708.00803.

[12] E. McClure, S. Berg, T. Armstrong, Geometric evolution as a source of discontinuous behavior in soft condensed matter, arXiv:1906.04073.

[13] A. Grassi, D. Morrison, Anomalies and the Euler characteristic of elliptic Calabi-Yau threefolds, Commun.Num.Theor.Phys. 6 (2012) 51-127 arXiv:1109.0042. 
[14] B. Green, String Theory on Calabi-Yau Manifolds, hep-th/9702155.

[15] A. Belhaj, J. Rasmussen, A. Sebbar, M. B. Sedra, On ADE Quiver Models and F-Theory Compactification, J.Phys. A39(2006)9339, hep-th/0512321.

[16] A. Akopyan, H. Edelsbrunner, The Weighted Mean Curvature Derivative of a SpaceFilling Diagram, Comput. Math. Biophys. 8 (2020) 51, arXiv:1908.06779.

[17] S. Franco, S. Gukov, S. Lee, R-K. Seong, J. Sparks, Lagrangian Disks in M-theory, JHEP11(2020)033, arXiv:1910.01645 [hep-th]

[18] V. Guillemin. A. Pollack, Differential Topology, Massachusetts Institute of Technology. Prentice-Hall, Inc., Englewood Clifts, New Jersey.

[19] V. G. Kac, Infinite dimensional Lie algebras, third edition, Cambridge University Press (1990).

[20] S. Franco and A. Hanany, Geometric dualities in $4 d$ field theories and their $5 d$ interpretation, JHEP 0304(2003)043, arXiv: hep-th/0207006.

[21] B. Feng, Y. H. He and F. Lam, On correspondences between toric singularities and (p,q)-webs, Nucl.Phys. B701 (2004) 334-356, arXiv:hep-th/0403133.

[22] S. Franco, A. Hanany and P. Kazakopoulos, Hidden exceptional global symmetries in $4 d$ CFTs, JHEP07 (2004) 060, arXiv:hep-th/0404065.

[23] C. Vafa, E. Witten, Dual String Pairs With $N=1$ And $N=2$ Supersymmetry In Four Dimensions, Nucl.Phys.Proc.Suppl.46 (1996)225, hep-th/9507050.

[24] E. Witten, String Theory Dynamics in Various Dimensions, Nucl. Phys. B443 (1995) 85, arXiv:hep-th/9503124.

[25] V. P. Frolov and R. Goswami, Surface geometry of 5D black holes and black rings. Physical Review D, 75(2007) 124001, arXiv:gr-qc/0612033.

[26] R. Emparan and H. S. Reall, A Rotating Black Ring Solution in Five Dimensions, Physical Review Letters, 10 (88) (2002) 101101, arXiv:hep-th/0110260

[27] A. Grassi, D. Morrison, Anomalies and the Euler characteristic of elliptic Calabi-Yau threefolds, arXiv:1109.0042.

[28] J. Polchinski, E. Witten, Evidence for Heterotic-Type I String Duality, Nucl.Phys.B460(1996)525.

[29] D. Cremades, L.E. Ibanez, F. Marchesano, Dynamical Supersymmetry Breaking in Standard-Like Models with Intersecting D6-Branes, Phys. Rev. D68 (2003)046002.

[30] R. Blumenhagen, L. Gorlich, B. Kors, Supersymmetric 4 D Orientifolds of Type IIA with D6-Branes at Angles, JHEP1(2000) 040. 